Sharif University of Technology
Scientia Iranica
SCIENTIA
I RAN I CA
http://scientiairanica.sharif.edu

\title{
The dependence of conditional spectra on the choice of target periods
}

\author{
A. Azarbakht* and A.R. Ghodrati \\ Department of Civil Engineering, Faculty of Engineering, Arak University, Arak, 38156-8-8349, Iran.
}

Received 26 May 2014; received in revised form 12 September 2015; accepted 18 April 2017

\author{
KEYWORDS \\ Conditional mean \\ spectrum; \\ Epsilon; \\ Eta; \\ Seismic hazard; \\ Disaggregation; \\ Next generation \\ attenuation; \\ Target period; \\ Return period; \\ Mean annual \\ frequency.
}

\begin{abstract}
The dependence of Conditional Mean Spectrum (CMS) and the corresponding standard deviation on different target period values were investigated in this paper by means of two types of target spectra, i.e. based on Epsilon and Eta indicators. The structural collapse capacities as well as the Mean Annual Frequency (MAF) of exceeding a limit state were taken into consideration. The results showed that the dependence of Eta-based CMS (ECMS) on the choice of target period was insensitive to the target period in the case of MAF calculation. However, this dependence was meaningfully low in the case of ECMS when compared to CMS in intensity-based ground motion selection. The Sum of the Squared Error (SSE) was utilized to compare different CMS cases. SSE was less in shorter return periods, e.g. 75 years, than in longer return periods, e.g. 2475 years. This dependence is also a function of choosing attenuation relationships. Therefore, four Next Generation Attenuation (NGA) relationships were employed in this study. In general, ECMS showed less dependence in all cases than the conventional CMS did.

(C) 2018 Sharif University of Technology. All rights reserved.
\end{abstract}

\section{Introduction}

Estimation of structural response, subjected to a probable future earthquake event, is usually performed based on nonlinear dynamic analysis. Therefore, selection of ground motion records is an important issue in dynamic response-history analysis. This selection is often done by choosing a set of records that have the most compatibility with a target spectrum [1], e.g. Uniform Hazard Spectrum (UHS). For clarity, the conventional UHS is achievable as written in Eq. (1) for an ideal site, in which future earthquake is characterized by a specific moment magnitude, distance, etc., i.e. in the case of using seismic hazard disaggregation results:

$$
\mu \ln S a(T i)=\mu \ln S a\left(M, R, T_{i}\right)+\varepsilon \sigma \ln S a(T i),
$$

\footnotetext{
*. Corresponding author.

E-mail address: a-azarbakht@araku.ac.ir (A. Azarbakht)
}

doi: $10.24200 /$ sci. 2017.4255 where $\mu \ln S a(T i)$ is the natural logarithm of spectral acceleration in an arbitrary period, $\mu \ln S a(M, R, T i)$ and $\sigma \ln S a(T i)$ are, respectively, the mean and standard deviation of the natural logarithm of spectral acceleration in an arbitrary period, and Epsilon $(\varepsilon)$ is defined as the number of standard deviations from the predicted value by an empirical ground motion model [1].

A sample UHS is shown in Figure 1 in the case of $M_{w}=7.2, R=11 \mathrm{~km}$ based on the CB08 attenuation model [2]. It is worth noting that previous researchers have revealed that employing UHS is usually conservative in the structural response estimation, especially at low probabilities in which UHS is too "broadband" (i.e., high amplitudes over an unrealistically broad range of periods) [1,3]. Therefore, obtaining an accurate prediction of structural response has been an important concern in recent years. The Conditional Mean Spectrum (CMS) was proposed in order to improve the deficiencies of UHS, i.e. to make it less conservative than the conventional UHS. CMS is obtained for a 


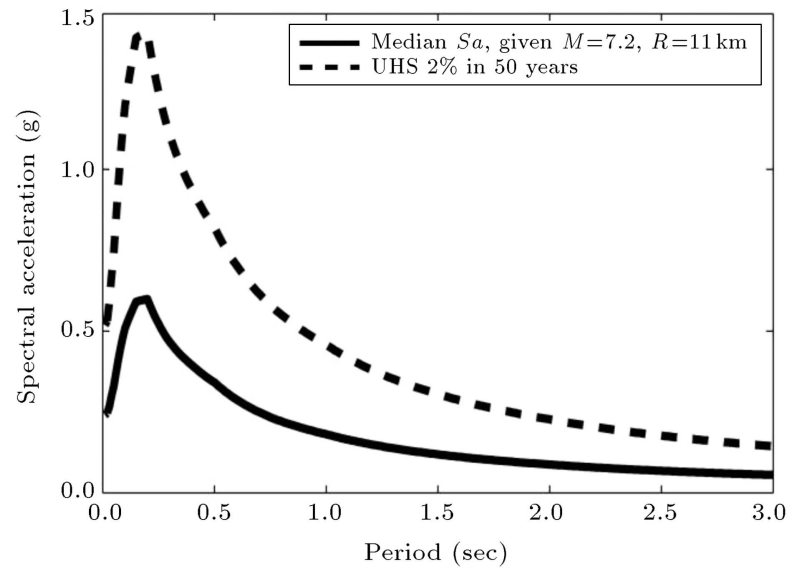

Figure 1. UHS and the median spectrum based on CB08 attenuation model for the case of $M=7.2$ and $R=11 \mathrm{~km}$.

specific site and the fundamental period of a given structure is discussed in details in the next section. Moreover, nonlinear response of a given structure is obviously a function of several periods, including the fundamental period. However, CMS only utilizes the fundamental period of a chosen structure and does not contain enough information about other periods. This dependence has been investigated in the cases of CMS and CS. The obtained results have shown that the choice of conditioning period for the CS can substantially impact structural response estimates in an intensity-based assessment, but risk-based assessments are relatively insensitive to the choice of conditioning period in the CS (given that the ground motions are carefully selected to ensure hazard consistency) $[4,5]$.

In this paper, the dependence of CMS, ECMS, CS, and ECS on the chosen target period is studied and the obtained results are comprehensively discussed. Therefore, a brief introduction to CMS is provided in the next section. Then, the dependence of different target spectra on the chosen target period is systematically investigated.

A brief introduction to the conditional mean spectra is provided in Section 2. Then, in Section 3, the influence of the given target period on conditional mean spectrum is investigated by means of CMS and ECMS. A sensitivity analysis is also performed in Section 4 in order to justify the period influence in different hazard levels. Section 5 is a sort of extension of Section 4 by implementing the conditional mean spectrum and its variations. As a final investigation, the influence of the target period on mean annual frequency of collapse is investigated in Section 6. Then, the concluding remarks are drawn.

\section{Conditional mean spectrum}

Conditional Mean Spectrum has been proposed in the literature to be employed as a target spectrum [1]. It is mathematically written as:

$$
\begin{gathered}
\mu \ln S a\left(T_{i}\right) \mid \ln S a\left(T^{*}\right)=\mu \ln S a\left(M, R, T_{i}\right) \\
+\rho\left(T_{i}, T^{*}\right) \varepsilon\left(T^{*}\right) \sigma \ln S a\left(T_{i}\right),
\end{gathered}
$$

where $T^{*}$ is the target period and $\rho\left(T_{i}, T^{*}\right)$ is the correlation coefficient between $\varepsilon\left(T_{i}\right)$ and $\varepsilon\left(T^{*}\right)$ as mathematically written in Eq. (3), i.e. for $T_{1}$ and $T_{2}$ as arbitrary periods. $\varepsilon\left(T^{*}\right)$ is obtained based on disaggregation analysis [1] of seismic hazard in a specific level of intensity measure. The other parameters in Eq. (2) have the same definition as illustrated in Eq. (1).

$\rho\left(\varepsilon\left(T_{1}\right), \varepsilon\left(T_{2}\right)\right)=$

$$
\frac{\sum_{k=1}^{n}\left(\varepsilon k\left(T_{1}\right)-\overline{\varepsilon\left(T_{1}\right)}\right)\left(\varepsilon k\left(T_{2}\right)-\overline{\varepsilon\left(T_{2}\right)}\right)}{\sqrt{\sum_{k=1}^{n}\left(\varepsilon k\left(T_{1}\right)-\overline{\varepsilon\left(T_{1}\right)}\right)^{2} \sum_{k=1}^{n}\left(\varepsilon k\left(T_{2}\right)-\overline{\varepsilon\left(T_{2}\right)}\right)^{2}}}
$$

where $\rho\left(\varepsilon\left(T_{1}\right), \varepsilon\left(T_{2}\right)\right)$ is the correlation coefficient between the Epsilons at $T_{1}$ and $T_{2}$ periods; $\varepsilon k\left(T_{1}\right)$ and $\varepsilon k\left(T_{2}\right)$ are the $k$ th data among the Epsilons, respectively, at $T_{1}$ and $T_{2}$ periods; $\overline{\varepsilon\left(T_{1}\right)}$ and $\overline{\varepsilon\left(T_{1}\right)}$ are, respectively, mean Epsilons at $T_{1}$ and $T_{2}$ periods; and $N$ is the number of ground motion records that are implemented. As an alternative to Eq. (3), a closedform solution is also available in order to obtain the correlation coefficients [6].

Implementing CS in practice and its related issues, such as selection of conditioning period, i.e. the minimum number of CMS cases which are necessary to cover the range of UHS; how to broaden CMS to match UHS with fewer conditioning periods; how to compute a CMS for several ground motion prediction equations that is consistent with probabilistic seismic-hazard analysis; the application of correlation coefficients to hard-rock sites; and the robustness of correlation coefficients, have been discussed by the previous research [7]. UHS-compatible Conditional Mean Spectra (CMS) and UHS spectra were developed to bridge the gap between deterministic and probabilistic seismic hazard evaluations, and used to evaluate liquefaction, lateral spreading, and settlement potential. The computed response was more consistent with the historical and geologic records than the UHS spectrum was [8]. The advantages and disadvantages of CMS are summarized in Table 1.

As it is obvious in Eq. (2), Epsilon is the key parameter in the development of CMS and it contains information from the elastic spectral shape of the considered record. On the other hand, a new parameter, named Eta, has been proposed in literature in order to improve the conventional Epsilon efficiency $[9,10]$. The Peak Ground Velocity (PGV) has been utilized to 
Table 1. Advantages and disadvantages of CMS.

\begin{tabular}{ll}
\hline \multicolumn{1}{c}{ Advantages } & \multicolumn{1}{c}{ Disadvantages } \\
\hline M, R, and Epsilon are employed to obtain CMS. & CMS is less available than UHS to engineers. \\
CMS amplitude is usually less than UHS amplitude. & \\
$\begin{array}{l}\text { Hence, if CMS is not un-conservative, then, } \\
\text { it can be claimed that it is a kind of }\end{array}$ & $\begin{array}{l}\text { CMS is site-specific. In } \\
\text { other words, it should be obtained } \\
\text { more optimum target spectrum compared with }\end{array}$ \\
sonventional UHS spectra. & separately for different sites. \\
& $\begin{array}{l}\text { CMS is dependent on target period. } \\
\text { For a specific structure which is }\end{array}$ \\
& sensitive to several periods, several \\
CMSs in several target periods & should be obtained. \\
\hline
\end{tabular}

define the Eta indicator. The Eta indicator is defined as mathematically written in Eq. (4):

$$
\eta=0.472+2.730 \varepsilon_{S a}-2.247 \varepsilon_{\mathrm{PGV}}
$$

where $\varepsilon_{S a}$ is the conventional Epsilon based on spectral acceleration and $\varepsilon_{\mathrm{PGV}}$ is Epsilon based on PGV, which is defined as written in Eq. (5):

$$
\varepsilon_{\mathrm{PGV}}=\frac{1}{2.247}\left(0.472+1.730 \varepsilon_{S a}^{\mathrm{target}}\right)
$$

Eta-based Conditional Mean Spectrum (ECMS) is obtained in a completely similar way to the conventional CMS framework as written in Eq. (6):

$$
S a(T)=\exp \left(\mu_{\ln S a(T)}+\eta^{\text {target }} \sigma_{\ln S a(T)} \rho_{(\eta(T), \eta(T *))}^{\prime}\right) .
$$

The target Eta and the correlation coefficient between the target Eta and an arbitrary period are, respectively, defined in Eqs. (7) and (8):

$$
\begin{aligned}
& \eta(t)=\eta^{\text {target }} \times \rho\left(\eta(T), \eta\left(T^{*}\right)\right), \\
& \rho^{\prime}\left(\eta(T), \eta\left(T^{*}\right)\right)=\frac{\rho\left(\eta(T), \eta\left(T^{*}\right)\right)+1.73}{2.730} .
\end{aligned}
$$

The mean and standard deviation of the natural logarithm of spectral acceleration are obtained by employing an appropriate ground motion prediction equation, e.g. CB08 in this study. The target earthquake scenario characteristics are obtained based on disaggregation analysis, e.g. by using the USGS tool in some prespecified periods [11]. The target period obviously plays an important role in the CMS calculation, which motivated the authors to focus on more comprehensively in the following sections.

\section{Dependence of CMS and ECMS on the selection of target period}

The first step in CMS calculation is to choose an appropriate target period for the conditioning purpose. Selection of this target period is a serious challenge since it affects the CMS shape significantly. As a consequence, any meaningful change in CMS shape can result in a different record selection and, obviously, different structural nonlinear response. In the case of multi-degree-of-freedom structures, several periods influence the structural nonlinear response, which means that there are several choices for the target period. The first natural period is usually selected as the target period since it dominates the structural deformation response $[12,13]$. On the other hand, other structural responses, such as base shear, floor acceleration, etc. are also more sensitive to other periods than to the first natural period [4,5]. Elimination of higher mode effects, in the selection of target period, results in the non-conservative estimation of structural response. To deal with this problem, a set of CMSs is needed to be calculated for a set of structural periods. Therefore, several records should be selected for each CMS; hence, the computational efforts will increase significantly. In addition, each CMS is representative of one structure period, which means different structure periods are not simultaneously taken into account. This issue is more problematic in the case that more than one structure are of interest to be analysed. The computational efforts in this case would increase in a way that using UHS is preferable. To clarify the exposition, a numerical example is given here to more elaborate on this issue. An ideal site, namely, the Riverside site in California State, is considered, which is located in $117.396 \mathrm{~W}, 33.953 \mathrm{~N}$. The following assumptions are also taken into account in order to use the CB08 attenuation model: 


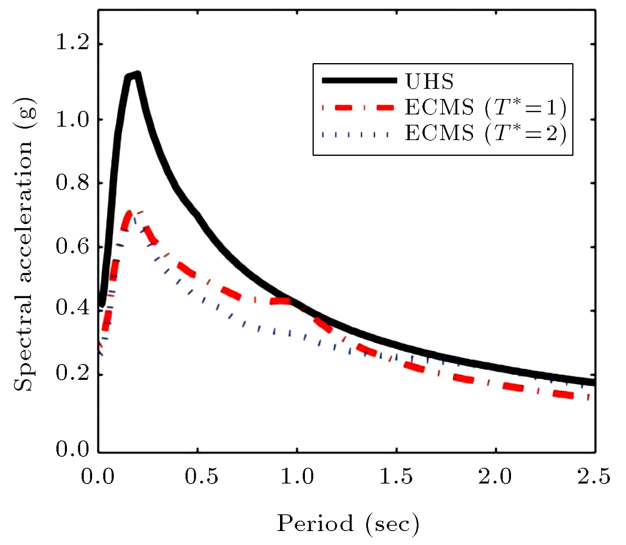

(a)

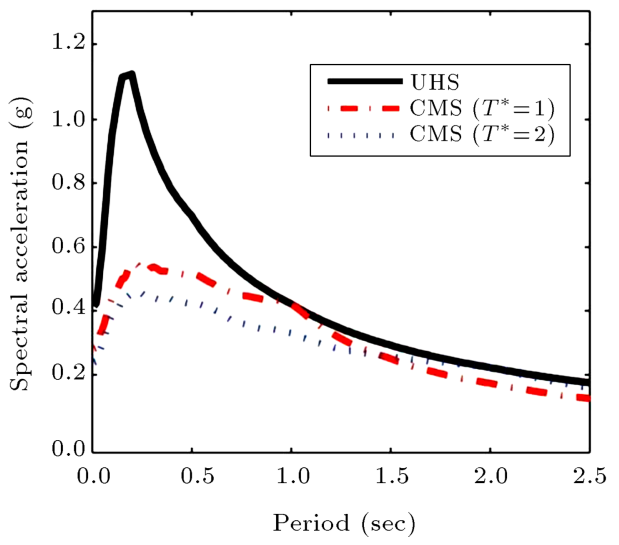

(b)

Figure 2. UHS and conditional mean spectrum at $T^{*}=1 \mathrm{sec}$ and $T^{*}=2 \mathrm{sec}$ in the cases of (a) Eta and (b) epsilon.

- Rupture angle $=35$ degrees;

- Rupture depth $=0 \mathrm{~km}$;

- Mean rupture angle $=90$ degrees;

- Mean shear wave velocity in the upper $30 \mathrm{~m}=$ $760 \mathrm{~m} / \mathrm{s}$;

- The depth corresponding to $2.5 \mathrm{~km} / \mathrm{s}$ velocity (z2.5) $=2.5 \mathrm{~km}$.

The disaggregation results for the Riverside site provide $M=7.4, R=19.8 \mathrm{~km}$, and the target Epsilon equal to 1.73 in the case of 2475 years of return period. Two arbitrary target periods are taken equal to $1 \mathrm{sec}$ and 2 sec. By using the pre-mentioned parameters and an appropriate ground motion prediction equation, i.e. CB08 in this study, the spectral acceleration is equal to $0.13 \mathrm{~g}$ and $0.07 \mathrm{~g}$, respectively, in the cases of $T^{*}=$ $1 \mathrm{sec}$ and $T^{*}=2 \mathrm{sec}$. The corresponding standard deviations are 0.66 and 0.68 , respectively, in the cases of $T^{*}=1 \mathrm{sec}$ and $T^{*}=2 \mathrm{sec}$. Finally, CMSs for the conditioning periods equal to $1 \mathrm{sec}$ and $2 \mathrm{sec}$ are shown in Figure 2(a) and (b), respectively, in the cases of ECMS and CMS.

As seen in Figure 2(b), CMS is sensitive to the choice of the target period. Additionally, it is intuitively obvious that ECMS is less sensitive to the target period, at least within the example context, than the obtained CMS is. To more elaborate on this issue, the sensitivity of CMS and ECMS to the target period is systematically investigated in the following section.

\section{Sensitivity of CMS and ECMS to the choice of target period}

In order to investigate the sensitivity of CMS and ECMS to the choice of target period, four levels of seismic hazard (in terms of return period) were taken into account, namely, 72, 200, 475, and 2475 years of return period. Disaggregation analysis of the Riverside site was performed for the four pre-mentioned return periods in the cases of spectral acceleration at periods equal to $0.1,0.2,0.3,0.5,1,2,3,4$, and $5 \mathrm{sec}$ [11]. The results of the disaggregation analysis are shown in Table 2.

The CMS curve was calculated for each hazard level, which resulted in nine different spectra for the nine target periods, as seen in Figure 3(a), in the case of 72 years of return period. The average of the nine spectra was then calculated, which obtained a new spectrum, called a benchmark-spectrum hereafter, as seen in Figure 3(b). The authors made this decision in order to define a point of comparison in this study. The same procedure was utilized in the ECMS case, to which the corresponding results are shown in Figure 3(c) and (d). It is worth noting that UHS always has the highest amplitude when compared to CMS and ECMS; this describes why UHS is a kind of conservative design spectrum. Additionally, as seen in Figure 3(a), the benchmark-spectrum has a meaningful difference with those in single CMS cases, whereas this difference is less in the case of ECMS, which is shown in Figure 3(c).

The benchmark-spectrum contains information from all chosen target periods, which makes it suitable to be the point of comparison in this study. In other words, each CMS, which has minimum deviation from the corresponding benchmark-spectrum, is less sensitive to the target period. To clarify, error terms were defined as written in Eqs. (9) and (10) in the cases of CMS and ECMS, respectively. A schematic description of the error terms is also shown in Figure 4.

$$
\begin{aligned}
& E_{\mathrm{CMS}}^{\left(T^{*}\right)}=100 \\
& * \frac{\left|\int_{0}^{T_{0}} S_{a, n}^{\mathrm{CMS}}\left(T, \xi \mid T^{*}\right) d T-\int_{0}^{T_{0}} \overline{S_{a, n}^{\mathrm{CMS}}\left(T, \xi \mid T^{*}\right)} d T\right|}{\int_{0}^{T_{0}} \overline{S_{a, n}^{\mathrm{CMS}}\left(T, \xi \mid T^{*}\right)} d T},
\end{aligned}
$$


Table 2. The results of the disaggregation analysis in the cases of $72,200,475$, and 2475 years of return periods versus different target periods.

\begin{tabular}{|c|c|c|c|c|c|c|c|c|c|}
\hline \multicolumn{10}{|c|}{$\begin{array}{l}2 \% \text { exceedance probability in } 50 \text { years or mean return time of } \\
2475 \text { years }\end{array}$} \\
\hline$T^{*}$ & 0.1 & 0.2 & 0.3 & 0.5 & 1 & 2 & 3 & 4 & 5 \\
\hline$M$ & 11 & 6.86 & 7 & 1.10 & & & & & \\
\hline$R(\mathrm{~km})$ & & & 16.2 & 17.4 & & & & & 5.4 \\
\hline Eps. & 1.06 & 1.67 & 1.68 & 1.7 & 1.7 & & & & 1.6 \\
\hline \multicolumn{10}{|c|}{$\begin{array}{l}10 \% \text { exceedance probability in } 50 \text { years or mean return time of } \\
475 \text { years }\end{array}$} \\
\hline$T^{*}$ & 01 & $0 ?$ & 0.3 & 0.5 & 1 & 2 & 3 & 4 & 5 \\
\hline$M$ & & & 7.02 & 7.14 & & & & & 56 \\
\hline$R(\mathrm{~km})$ & 1 & 18 & 19 & 20 & & & & & .4 \\
\hline Eps. & 1.21 & 1.22 & 1.22 & 1.21 & & & & & 1.02 \\
\hline \multicolumn{10}{|c|}{$\begin{array}{l}22 \% \text { exceedance probability in } 50 \text { years or mean return time of } \\
200 \text { years }\end{array}$} \\
\hline$T^{*}$ & & 0.2 & 3 & 0.5 & 1 & 2 & 3 & 4 & 5 \\
\hline$M$ & & & 6.99 & 7.1 & & 7. & & & .48 \\
\hline$R(\mathrm{~km})$ & 19 & & 20.7 & 21.6 & & & & & 27.9 \\
\hline Eps. & 0.89 & 0.89 & 0.88 & 0.87 & 0.83 & 0.74 & 0.65 & 0.62 & 0.63 \\
\hline \multicolumn{10}{|c|}{$\begin{array}{l}50 \% \text { exceedance probability in } 50 \text { years or mean return time of } \\
\qquad 2 \text { years }\end{array}$} \\
\hline$T^{*}$ & 0.1 & 0.2 & 0.3 & 0.5 & 1 & 2 & 3 & 4 & 5 \\
\hline$M$ & 6.7 & 6.8 & 6.91 & 7.01 & 7. & 7.27 & 7.33 & & 7.35 \\
\hline$R(\mathrm{~km})$ & 21 & 22 & 23.7 & 25.1 & 28 & 35 & & 32.9 & 34.9 \\
\hline Eps. & 0.39 & 0.38 & 0.39 & 0.37 & 0.33 & 0.24 & 0.14 & 0.1 & 0.14 \\
\hline
\end{tabular}

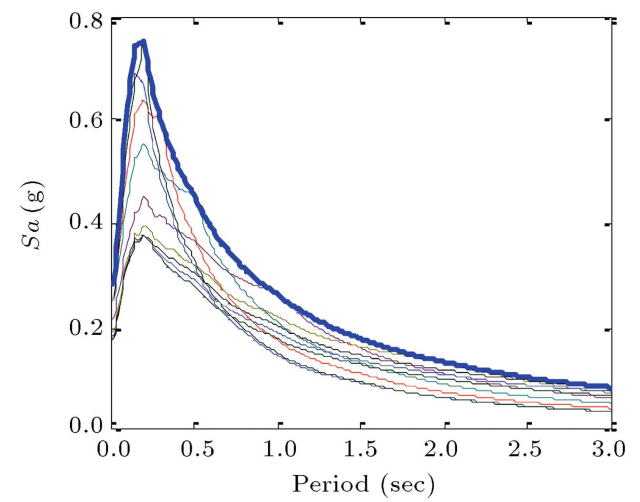

(a)

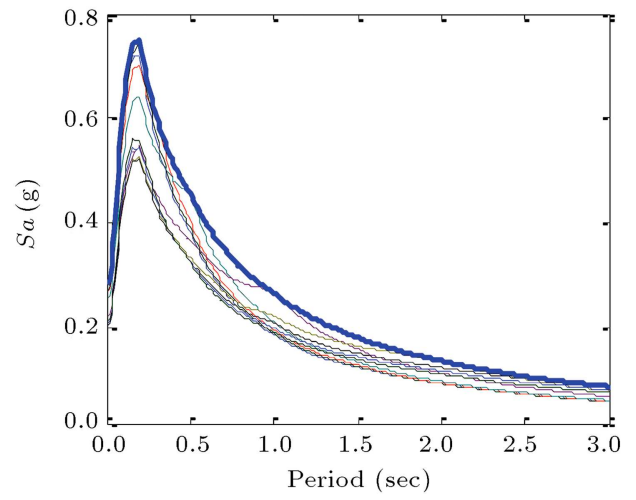

(c)

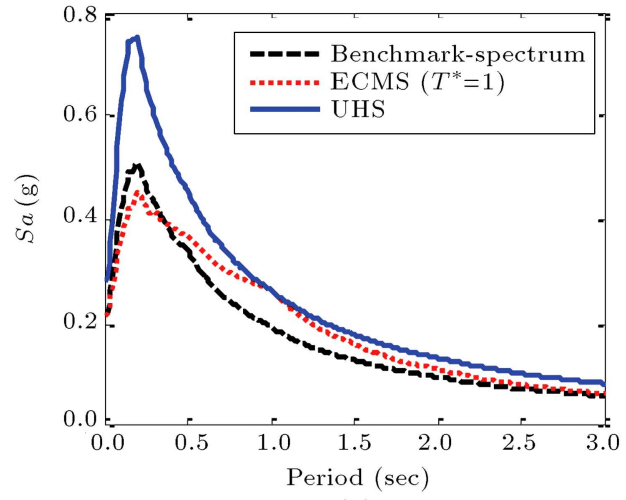

(b)

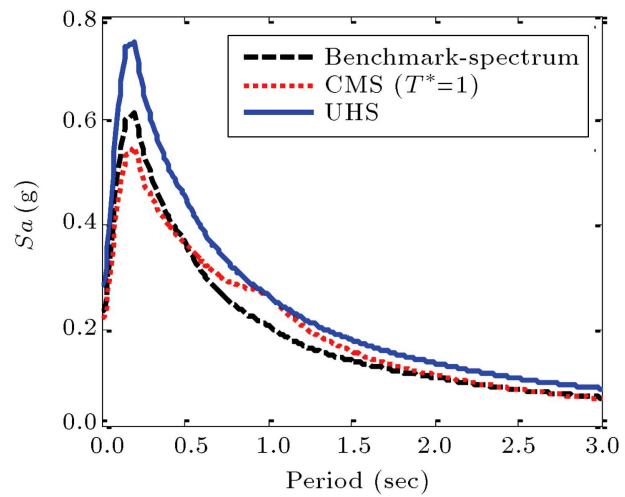

(d)

Figure 3. UHS and individual CMS cases for different target periods: (a) CMS and (c) ECMS; UHS and benchmark-spectrum in the cases of (b) CMS and (d) ECMS. 


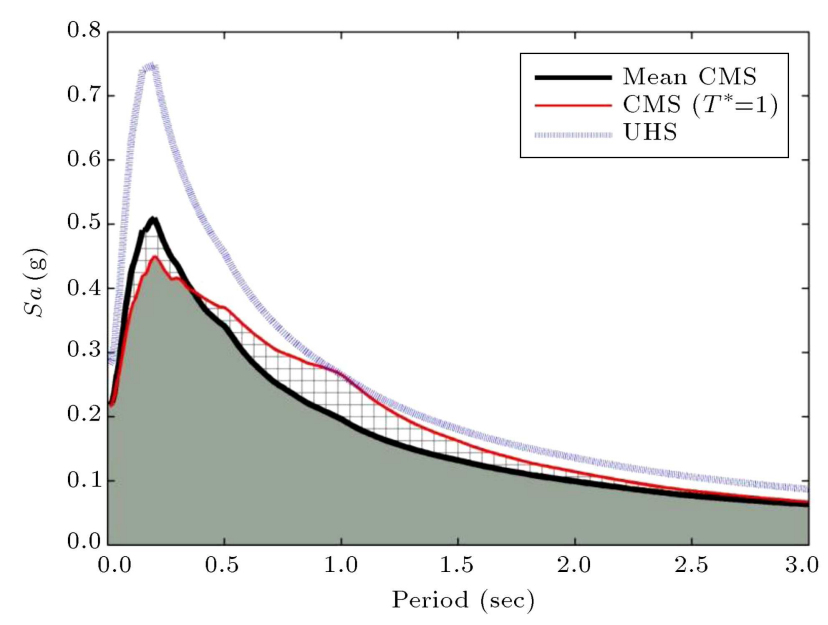

Figure 4. Schematic description of error terms as defined in Eqs. (9) and (10).

$$
\begin{aligned}
& E_{\mathrm{ECMS}}^{\left(T^{*}\right)}=100 \\
& * \frac{\left|\int_{0}^{T_{0}} S_{a, n}^{\mathrm{ECMS}}\left(T, \xi \mid T^{*}\right) d T-\int_{0}^{T_{0}} \overline{S_{a, n}^{\mathrm{ECMS}}\left(T, \xi \mid T^{*}\right)} d T\right|}{\left.\int_{0}^{T_{0}} \overline{S_{a, n}^{\mathrm{ECMS}}\left(T, \xi \mid T^{*}\right.}\right)} d T
\end{aligned}
$$

where $n$ is the number of CMS cases, $T^{*}$ is the target period, $\xi$ is the critical damping ratio, and $T_{0}$ is the upper bound of the considered period for the error integration. $S_{a, n}^{\mathrm{CMS}}$ and $S_{a, n}^{\mathrm{ECMS}}$ are the $n$th spectral ordinates in the cases of CMS and ECMS, respectively. $\overline{S_{a, n}^{\mathrm{CMS}}}$ and $\overline{S_{a, n}^{\mathrm{ECMS}}}$ are benchmark-spectra in the cases of CMS and ECMS, respectively.

The error was calculated based on Eqs. (9) and (10) for different attenuation models, namely, CY08 [14], CB08 [2], AS08 [15], and BA08 [16], and for different hazard levels versus target periods as shown in Figure 5(a) to (d).

As seen in Figure 5, the error value increases by increase in the hazard level for both CMS and ECMS cases, i.e. longer return periods have higher errors. In other words, the error value in the case of 2475 years of return period is approximately three times the corresponding error in a 72-year return period. Additionally, different ground motion prediction models can result in different amounts of error. For example, BA08 and CY08 have lower error values than the CB08 and AS08 cases. It means that the conditional spectra, which have employed the BA08 or CY08 model, have less sensitivity to the target period than the rest of ground motion prediction models.

For all four hazard levels as well as all target periods, the Eta based CMS has less error than the corresponding Epsilon-based CMS has. However, in all cases, the obtained error decreases by increase in the target period. The highest error value (44 and 31 percent, respectively, in the cases of CMS and ECMS) corresponds to the case with $T^{*}=0.1 \mathrm{sec}, \mathrm{CB} 08$ model, and 2475 years of return period. On the other hand, the lowest error value ( 9 and 8 percent, respectively, in the cases of CMS and ECMS) corresponds to the case with $T^{*}=0.1 \mathrm{sec}, \mathrm{CY} 08$ model, and 75 years of return period.

\section{Sensitivity of CMS, ECMS, and the corresponding variations to the choice of target period}

CMS and its variations, i.e. CMS, CMS+Sigma, and CMS-Sigma, were simultaneously taken into account in this section in order to investigate the error. For this purpose, the same definition of error was employed for CMS, CMS+Sigma and CMS-Sigma curves. Then, the average error of the three cases was calculated and taken as the final error index. The calculated error in the case of CMS (as discussed in the previous section) and the error based on the CMS and its variations are presented in Table 3 versus the target period for

Table 3. The results of error calculation in the cases of $72,200,475$, and 2475 years of return period versus different

\begin{tabular}{|c|c|c|c|c|c|c|c|c|c|c|}
\hline Return period & Target spectrum & $0.1 \mathrm{sec}$ & $0.2 \mathrm{sec}$ & $0.3 \mathrm{sec}$ & $0.5 \mathrm{sec}$ & $1 \mathrm{sec}$ & $2 \mathrm{sec}$ & $3 \mathrm{sec}$ & $4 \mathrm{sec}$ & $5 \mathrm{sec}$ \\
\hline \multirow[t]{2}{*}{$2475 \mathrm{yrs}$} & CMS & 0.44 & 0.43 & 0.33 & 0.21 & 0.22 & 0.3 & 0.32 & 0.31 & 0.27 \\
\hline & $\mathrm{CMS}+/-$ std & 0.46 & 0.45 & 0.35 & 0.23 & 0.27 & 0.37 & 0.39 & 0.37 & 0.33 \\
\hline \multirow[t]{2}{*}{$475 \mathrm{yrs}$} & CMS & 0.33 & 0.32 & 0.24 & 0.15 & 0.17 & 0.21 & 0.23 & 0.23 & 0.2 \\
\hline & $\mathrm{CMS}+/-$ std & 0.36 & 0.35 & 0.28 & 0.2 & 0.23 & 0.26 & 0.29 & 0.3 & 0.28 \\
\hline \multirow[t]{2}{*}{$200 \mathrm{yrs}$} & CMS & 0.25 & 0.24 & 0.18 & 0.12 & 0.13 & 0.16 & 0.17 & 0.18 & 0.16 \\
\hline & $\mathrm{CMS}+/-\mathrm{std}$ & 0.32 & 0.31 & 0.28 & 0.22 & 0.22 & 0.21 & 0.25 & 0.27 & 0.26 \\
\hline \multirow[t]{2}{*}{$72 \mathrm{yrs}$} & CMS & 0.15 & 0.14 & 0.11 & 0.09 & 0.07 & 0.11 & 0.11 & 0.1 & 0.12 \\
\hline & $\mathrm{CMS}+/-$ std & 0.34 & 0.33 & 0.3 & 0.26 & 0.23 & 0.21 & 0.23 & 0.25 & 0.27 \\
\hline
\end{tabular}
target periods in the case of CMS. 


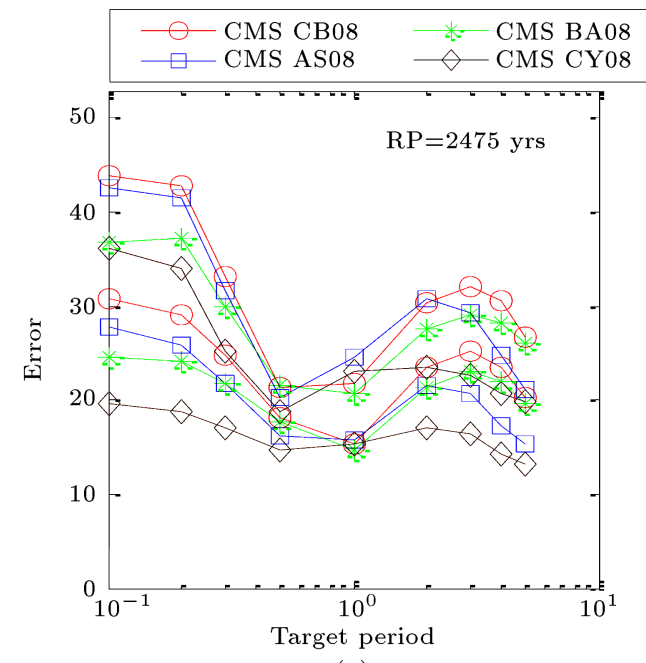

(a)

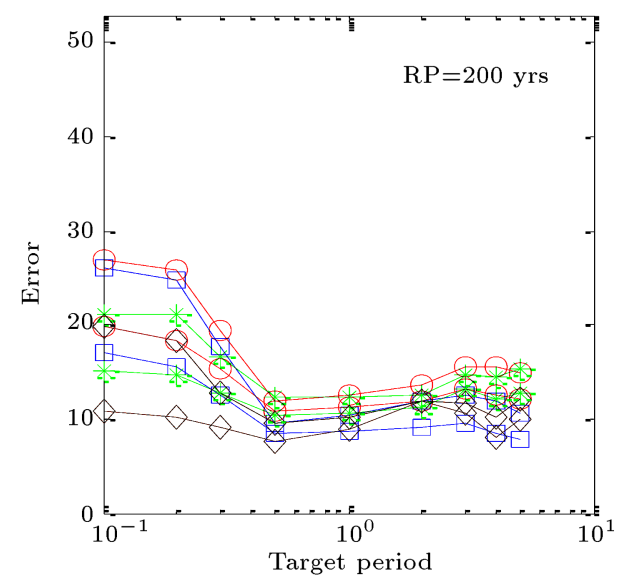

(c)

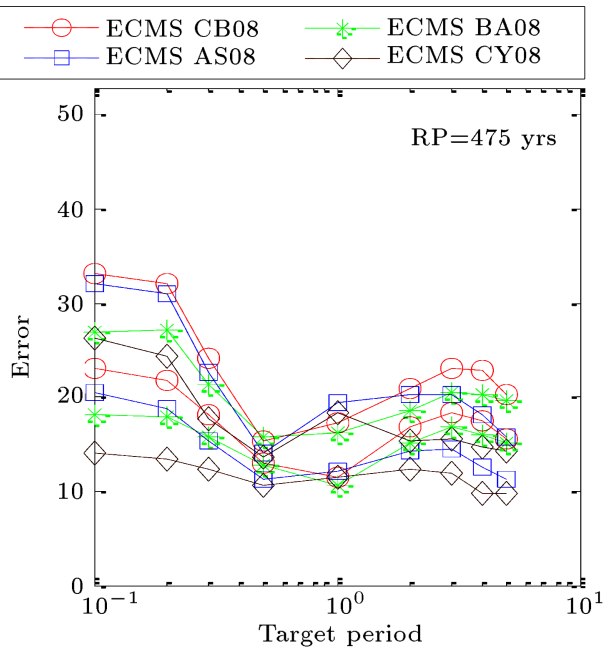

(b)

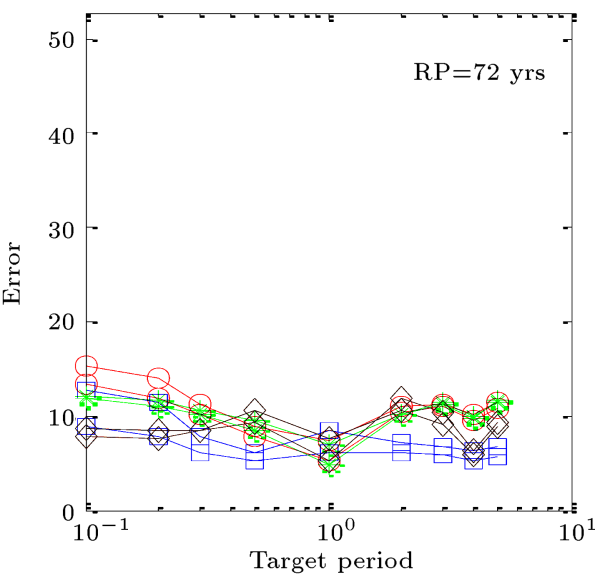

(d)

Figure 5. Errors for different attenuation models and different hazard levels versus target periods: (a) RP $=2475$ years, (b) $\mathrm{RP}=475$ years, (c) $\mathrm{RP}=200$ years, and (d) $\mathrm{RP}=72$ years.

different return periods. As seen in Table 3, in low hazard levels, e.g. 72 years of return period, the error is higher in the case that the CMS variations are taken into account. However, the difference between using CMS and its variations is not significant when high levels of hazard, e.g. 2475 years of return period, are employed.

The calculated error in the case of ECMS, as discussed in the previous section, and the error based on the ECMS and its variations are presented in Table 4 versus the target period for different return periods. The same trend to that in Table 3 is seen in Table 4. However, it is obvious that error amplitude decreases when ECMS is taken into account.

\section{Mean annual frequency of collapse as a function of target period}

The influence of the chosen target period on the shape of target spectra has been investigated in the previous sections. However, the record selection, the structural response, and the mean annual frequency of exceeding a given limit state are the final interests of engineering applications, which are still open. Therefore, record selection was performed in this section based on the compatibility of a set of records to the target spectra.

First, the target spectra were selected by means of five different cases including (1) UHS; (2) CMS; (3) ECMS; (4) CS, i.e. CMS and the corresponding variations; and (5) ECS, i.e. ECMS and the corresponding variations. All the considered cases corresponded to 2475 years of return period. In each selection process, 40 GMRs were selected out of a general set of GMRs being compatible with the target spectra in the range of $0.2 T^{*}$ to $2 T^{*}$. The general set of GMRs, which was used in this study, contained 530 GMRs that were available in [17]. The mean spectra of the 40 chosen GMRs are shown in Figure 6(a) and (b) for the cases of CMS and CS, respectively. By considering five different target spectra and nine different target periods, there were 45 different scenarios to be taken into account.

The given structure in this section was selected 
Table 4. The results of error calculation in the cases of $72,200,475$, and 2475 years of return period versus different target periods in the case of ECMS.

\begin{tabular}{|c|c|c|c|c|c|c|c|c|c|c|}
\hline Return period & Target spectrum & $0.1 \mathrm{sec}$ & $0.2 \mathrm{sec}$ & $0.3 \mathrm{sec}$ & $0.5 \mathrm{sec}$ & $1 \mathrm{sec}$ & $2 \mathrm{sec}$ & $3 \mathrm{sec}$ & $4 \mathrm{sec}$ & $5 \mathrm{sec}$ \\
\hline \multirow[t]{2}{*}{$2475 \mathrm{yrs}$} & ECMS & 0.31 & 0.29 & 0.25 & 0.18 & 0.15 & 0.24 & 0.25 & 0.23 & 0.2 \\
\hline & ECMS+/-std & 0.33 & 0.31 & 0.27 & 0.2 & 0.19 & 0.28 & 0.31 & 0.29 & 0.26 \\
\hline \multirow[t]{2}{*}{$475 \mathrm{yrs}$} & ECMS & 0.23 & 0.22 & 0.18 & 0.13 & 0.11 & 0.17 & 0.18 & 0.18 & 0.16 \\
\hline & ECMS+/-std & 0.27 & 0.26 & 0.24 & 0.2 & 0.2 & 0.22 & 0.25 & 0.25 & 0.24 \\
\hline \multirow[t]{2}{*}{$200 \mathrm{yrs}$} & ECMS & 0.18 & 0.17 & 0.14 & 0.1 & 0.09 & 0.13 & 0.14 & 0.14 & 0.13 \\
\hline & ECMS+/-std & 0.28 & 0.27 & 0.25 & 0.22 & 0.21 & 0.2 & 0.22 & 0.24 & 0.23 \\
\hline \multirow[t]{2}{*}{$72 \mathrm{yrs}$} & ECMS & 0.13 & 0.12 & 0.1 & 0.08 & 0.05 & 0.1 & 0.11 & 0.1 & 0.11 \\
\hline & ECMS+/-std & 0.3 & 0.29 & 0.27 & 0.24 & 0.22 & 0.2 & 0.21 & 0.22 & 0.24 \\
\hline
\end{tabular}

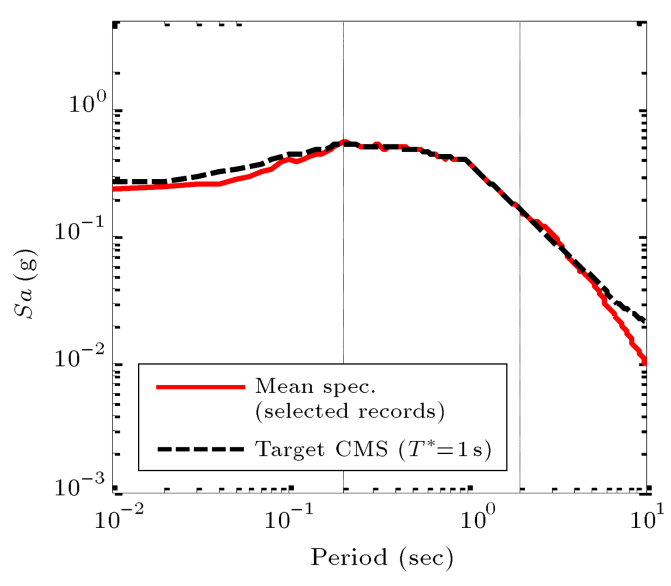

(a)

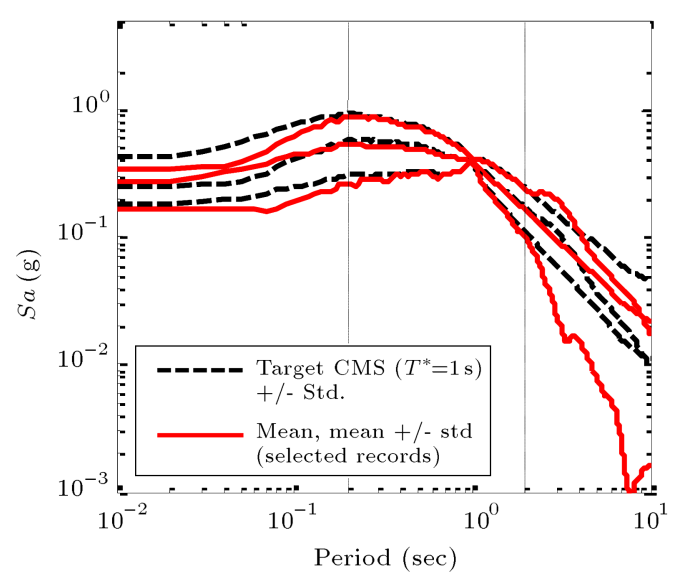

(b)

Figure 6. Record selection based on: (a) CMS and (b) CMS and its variations.

as the SPEAR building. The detailed characteristics can be found in [18]. The SPEAR building was a 3 -story 3D reinforced concrete structure designed by Fardis [19], for which a pseudo-dynamic experiment was performed at full scale at the ELSA Laboratory, within the European research project SPEAR ('Seismic performance assessment and rehabilitation of existing buildings') [18]. The structure had $T_{1}=0.85 \mathrm{sec}$. A more detailed explanation of the model and comparison of experimental and numerical results can be found in $[20]$.

The response of the given structure was calculated by means of incremental dynamic analysis [21]. The last point of each IDA curve was assumed to be a reasonable indicator of global instability, which is interpreted as the collapse capacity in this paper. The median collapse capacity versus different target periods is shown in Figure 7(a) in the cases of CMS and ECMS, and in Figure 7(b) in the cases of CS and ECS. As seen in Figure 7(a) and (b), the Eta based median collapse capacity is a little higher than the Epsilon based median collapse capacity, especially when the variations of the target spectrum are taken into consideration. This is an evidence that the conventional Epsilon based target spectrum can result in underestimation of structural demand [22].

The MAFs of collapse due to $S_{a}(T=1 \mathrm{sec})=$ $x$ are shown in Figure 8 for all different five target spectra. As seen in Figure 8, the highest MAF value is of UHS as expected. However, the Epsilon-based target spectra as well as Eta-based target spectra have lower MAF values. To clarify, the MAF values are summarized in Table 5 for all 45 scenarios corresponding to 2475 years of return period. It is obvious that the MAF values are influenced by the target spectra in the record selection approach. However, the results obtained 
Table 5. MAF results for different target periods and different target spectra.

\begin{tabular}{cccccccccc}
\hline Target period & $\mathbf{0 . 1} \mathbf{~ s e c}$ & $\mathbf{0 . 2} \mathbf{~ s e c}$ & $\mathbf{0 . 3} \mathbf{~ s e c}$ & $\mathbf{0 . 5} \mathbf{~ s e c}$ & $\mathbf{1} \mathbf{~ s e c}$ & $\mathbf{2} \mathbf{s e c}$ & $\mathbf{3} \mathbf{s e c}$ & $\mathbf{4} \mathbf{~ s e c}$ & $\mathbf{5} \mathbf{s e c}$ \\
\hline UHS & $8.00 \mathrm{e}-04$ & $9.81 \mathrm{e}-04$ & 0.0015 & $9.78 \mathrm{e}-04$ & $6.77 \mathrm{e}-04$ & $5.13 \mathrm{e}-04$ & $4.30 \mathrm{e}-04$ & $5.29 \mathrm{e}-04$ & $3.89 \mathrm{e}-04$ \\
CMS & $5.57 \mathrm{e}-04$ & $1.29 \mathrm{e}-04$ & $3.19 \mathrm{e}-04$ & $2.48 \mathrm{e}-04$ & $4.82 \mathrm{e}-04$ & $2.98 \mathrm{e}-04$ & $2.45 \mathrm{e}-04$ & $2.00 \mathrm{e}-04$ & $2.38 \mathrm{e}-04$ \\
ECMS & $7.99 \mathrm{e}-04$ & $1.55 \mathrm{e}-04$ & $4.26 \mathrm{e}-04$ & $2.77 \mathrm{e}-04$ & $4.55 \mathrm{e}-04$ & $3.04 \mathrm{e}-04$ & $2.25 \mathrm{e}-04$ & $1.97 \mathrm{e}-04$ & $3.24 \mathrm{e}-04$ \\
CS & $1.00 \mathrm{e}-03$ & $5.36 \mathrm{e}-04$ & $6.90 \mathrm{e}-04$ & $7.94 \mathrm{e}-04$ & $5.00 \mathrm{e}-04$ & $2.66 \mathrm{e}-04$ & $2.85 \mathrm{e}-04$ & $2.17 \mathrm{e}-04$ & $2.63 \mathrm{e}-04$ \\
ECS & $1.20 \mathrm{e}-03$ & $4.16 \mathrm{e}-04$ & $5.54 \mathrm{e}-04$ & $7.48 \mathrm{e}-04$ & $5.69 \mathrm{e}-04$ & $2.75 \mathrm{e}-04$ & $2.64 \mathrm{e}-04$ & $2.17 \mathrm{e}-04$ & $2.54 \mathrm{e}-04$ \\
\hline
\end{tabular}

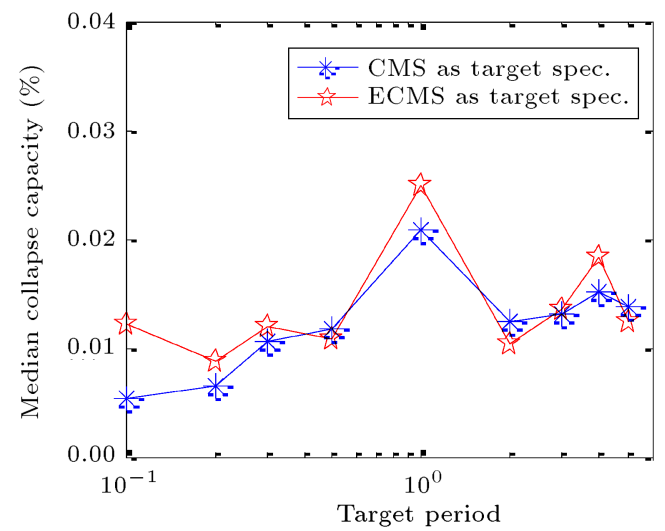

(a)

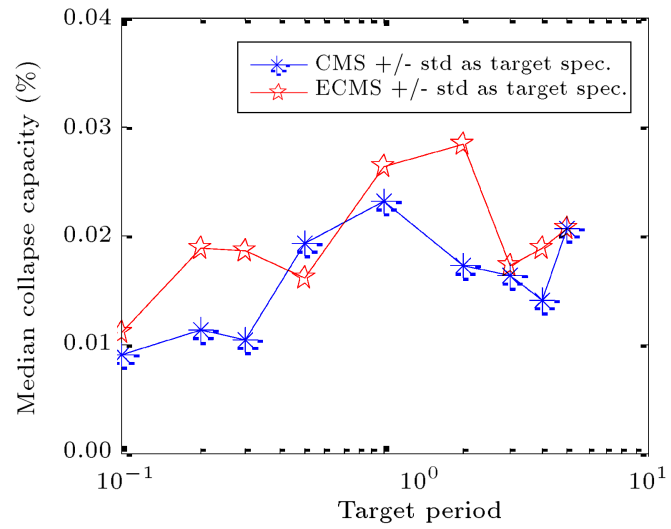

(b)

Figure 7. The median collapse capacity versus different target periods: (a) CMS and ECMS and (b) CS and ECS.

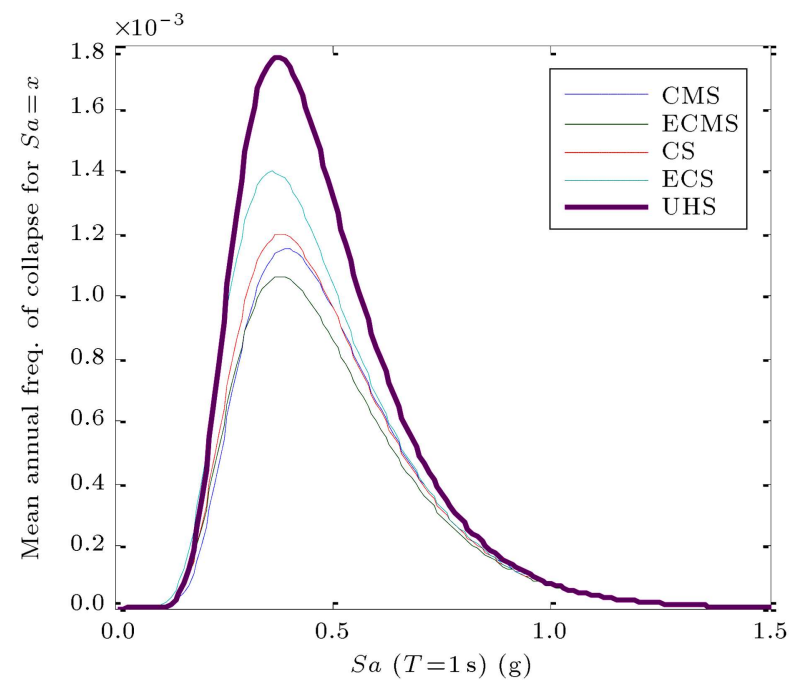

Figure 8. The MAF of collapse due to $S_{a}(T=1 \mathrm{sec})=x$ for different scenarios for target spectra.

based on CMS and ECMS as well as the results for CS and ECS are in agreement. This conclusion was also confirmed in the previous research, e.g. $[4,5]$.

\section{Conclusion}

The conditional mean spectrum had been introduced as an alternative to the conventional UHS. However, some issues were still necessary to be investigated about these kinds of new target spectra. One important issue was the dependence of the conditional mean spectrum on the selection of the target period, which was investigated in this paper. This issue is important especially in the case of multi-degree-of-freedom systems that are sensitive to more than one period.

45 different scenarios were taken into account by means of different target periods, different hazard levels, and different target spectra. The results showed that this dependence was negligible between CMS and ECMS as well as between CS and ECS when MAF calculation was of interest. However, in the case of intensity-based ground motion selection, this dependence was meaningful in the case that very short periods or very long periods were selected as target periods with high levels of seismic hazard. Generally speaking, the Eta-based CMS showed less dependence than the conventional Epsilon based CMS in the case of intensity-based ground motion selection. Therefore, as a conclusion, by employing ECMS in low levels of seismic hazard, the dependence of target spectra on the selection of target period was reduced. However, the results are limited to the context of this paper; hence, more investigations are still open for future research.

The choice of attenuation model also has influence on this dependence. Four NGA attenuation models, i.e. $\mathrm{AS} 08, \mathrm{BA} 08, \mathrm{CY} 08$, and CB08, were taken into consideration in this paper. The results revealed that CY08 and CB08 respectively corresponded to the minimum and maximum amounts of this dependence. 


\section{References}

1. Baker, J.W. "Conditional mean spectrum: Tool for ground motion selection", ASCE Journal of Structural Engineering, 173(3), pp. 322-331 (2011).

2. Campbell, K.W. and Bozorgnia, Y. "NGA ground motion model for the geometric mean horizontal component of PGA, PGV, PGD and 5\% damped linear elastic response spectra for periods ranging from 0.01 to 10 s", Earthquake Spectra, 24(1), pp. 139-171 (2008).

3. Somerville, P.G. and Thio, H.K. "Development of ground motion time histories for seismic design", Proceedings of the Ninth Pacific Conference on Earthquake Engineering Building an Earthquake-Resilient Society, Auckland, New Zealand, 14-16 April (2011).

4. Lin, T., Haselton, C.B. and Baker, J.W. "Conditionalspectrum-based ground motion selection, Part I: Hazard consistency for risk-based assessments", Earthquake Engineering and Structural Dynamics, 42(12), pp. $1847-1865$ (2013).

5. Lin, T., Haselton, C.B. and Baker, J.W. "ConditionalSpectrum-based ground motion selection. Part II: Intensity-based assessments and evaluation of alternative target spectra", Earthquake Engineering and Structural Dynamics, 42(12), pp. 1867-1884 (2013).

6. Baker, J.W. and Jayaram, N. "Correlation of spectral acceleration values from NG A ground motion models", Earthquake Spectra, 24(1), pp. 299-317 (2008).

7. Carlton, B. and Abrahamson, N. "Issues and approaches for implementing conditional mean spectra in practice", Bulletin of the Seismological Society of America, 104(1), pp. 503-512 (2014).

8. Hashash, Y., Abrahamson, N., Olson, S., Hague, S. and Kim, B. "Conditional mean spectra in site-specific seismic hazard evaluation for a Major River Crossing in the Central united states", Earthquake Spectra, 31(1), pp. 47-69 (2014).

9. Mousavi, M., Ghafory-Ashtiany, M. and Azarbakht, A. "A new indicator of elastic spectral shape for the reliable selection of ground motion records", Earthquake Engineering and Structural Dynamics, 40(12), pp. 1403-1416 (2011).

10. Azarbakht, A., Shahri, M. and Mousavi, M. "Reliable estimation of the mean annual frequency of collapse by considering ground motion spectral shape effects", Bulletin of Earthquake Engineering, 13(3), pp. 777-797 (2015).

11. USGS. United State of Geological Survey, Available from: https://geohazards.usgs.gov/deaggint/2008/.

12. Bazzurro, P. and Cornell, C.A. "Seismic hazard analysis of nonlinear structures I: Methodology", $A S C E$ Journal of Structural Engineering, 120(11), pp. 33203344 (1994).

13. Cornell, C.A., Jalayer, F., Hamburteger, R.O. and Foutch, D.A. "Probabilistic basis for 2000 SAC federal emergency management agency steel moment frame guidelines", ASCE Journal of Structural Engineering, 128(4), pp. 526-533 (2002).

14. Chiou, B.S.J. and Youngs, R.R. "An NGA model for the average horizontal component of peak ground motion and response spectra", Earthquake Spectra, 24(1), pp. 173-215 (2008).

15. Abrahamson, N.A. and Silva, W.J. "Summary of the Abrahamson \& Silva NGA ground motion relations", Earthquake Spectra, 24(1), pp. 67-97 (2008).

16. Boore, D.M. and Atkinson, G.M. "Ground-Motion prediction equations for the average horizontal component of PGA, PGV, and 5\%-damped PSA at spectral periods between $0.01 \mathrm{~s}$ and $10.0 \mathrm{~s}$ ", Earthquake Spectra, 24(1), pp. 99-138 (2008).

17. Baker, J.W. and Cornell, C.A., Vector-Valued Ground Motion Intensity Measures for Probabilistic Seismic Demand Analysis, PEER Report 2006/08, Pacific Earthquake Engineering Research Center, University of California, Berkeley, CA (2006).

18. Negro, P., Mola, E., Molina, F.J. and Magonette, G.E. "Full-scale testing of a torsionally unbalanced threestory non-seismic RC frame", Proceedings of the 13th World Conference on Earthquake Engineering, Paper No. 968 (2004).

19. Fardis, M.N., Design of an Irregular Building for the SPEAR Project-Description of the 3-Storey Structure, University of Patras, Structures Laboratory, Greece (2002).

20. Fajfar, P., Dolsek, M., Marusic, D. and Stratan, A. "Pre- and post-test mathematical modeling of a plan-asymmetric reinforced concrete frame building", Earthquake Engineering and Structural Dynamics, 35(11), pp. 1359-1379 (2006).

21. Vamvatsikos, D. and Cornell, C.A. "Incremental dynamic analysis", Earthquake Engineering and Structural Dynamics, 31(3), pp. 491-514 (2002).

22. Mousavi, M., Shahri, M. and Azarbakht, A. "E_CMS: A new design spectrum for nuclear structure in high levels of seismic hazard", Nuclear Engineering and Design, 252, pp. 27-33 (2012).

\section{Biographies}

Alireza Azarbakht is Associate Professor at Arak University, Arak, Iran. His current research activities include performance-based earthquake engineering, ground motion prediction equations, conditional spectra, record selection for seismic analysis, and seismic performance of infrastructures. More details can be found in www.alireza.azarbakht.info

Ahmad Reza Ghodrati is a graduate student in the Civil Engineering Department at Arak University, Iran. His main research interest is performance-based earthquake engineering. 